\title{
SELECTED COGNITIVE AND EMOTIONAL RESOURCES OF UNTRAINED YOUTH AND YOUNG WRESTLERS
}

\author{
Emotional intelligence and creativity
}

\author{
KATARZYNA RUTKOWSKA ${ }^{1}$, DARIUSZ GIERCZUK ${ }^{2}$ \\ The Josef Pilsudski University of Physical Education in Warsaw, \\ Faculty of Physical Education and Sport in Biała Podlaska, \\ Department of Psychology ${ }^{1}$, Department of Sports Theory ${ }^{2}$
}

\author{
Mailing address: Katarzyna Rutkowska, Faculty of Physical Education and Sport, \\ 2 Akademicka Street, 21-500 Biała Podlaska, tel.: +48 83 3428779, fax: +48 83 3428800, \\ e-mail: kr@psychologsportu.pl
}

\begin{abstract}
Introduction. Wrestling is a sport that requires proper physical and mental preparation. The authors present selected psychological resources, emotional intelligence and creative attitude, as factors influencing the broadly defined mastery, both in the area of sport and out of it. These variables guarantee better adaptation to changing conditions and they are also connected with the effectiveness of handling different situations and optimal problem solving. The aim of the study was to diagnose emotional intelligence and creative attitude among young female and male wrestlers. Material and methods. The study included 20 females from the regional team of cadets and juniors and 19 male wrestlers from the sports school. The control group consisted of two deliberately selected groups of untrained students -20 girls and 19 boys. KANH and INTE questionnaires were used in the study. Results. In the three groups under investigation medium levels of emotional intelligence and creative vs. reproductive attitude were observed. It was only female wrestlers who manifested low levels of emotional intelligence which, similar to the levels of nonconformity, heuristic thinking and creative attitude, were lower than in female students from the control group. The female wrestlers demonstrated higher levels of conformity and reproductive attitude. Female students demonstrated higher levels of emotional intelligence and conformity than male students. No differences between male and female wrestlers were observed. Conclusions. The findings show that it is necessary to conduct longitudinal research on athletes and untrained individuals. Bearing in mind that learning a motoric task depends, inter alia, on intellectual and emotional factors, the authors also recommend another course of research, i.e. the verification of how these variables are related to the effectiveness of learning motoric tasks. The authors' own research results as well as further (planned) investigations will make it possible to profile various educational effects and deliberately strengthen the potential of young wrestlers.
\end{abstract}

Key words: emotional intelligence, creative attitude, wrestling

\section{Introduction}

Over the years, creativity has been defined in many different ways. At first associated with arts, it was later used in terms of the broadly defined culture. Creativity was also observed in human potential. There appeared theories that emphasized a natural human tendency for transgression and hubristic motivation $[1,2,3]$. Currently the notion of creative attitude is not limited only to innovation and the act or the process of creating. Contrary to reproductive attitude, creative attitude is a vital psychological resource since it boosts self-esteem and self-confidence, and it is connected with a number of universal features that are useful in various activities. These features include, charisma, diligent acting aimed at achieving specific goals, ability to adapt as well as constant development and improvement $[3,4,5,6]$.

Despite initial misgivings, currently a number of investigations emphasise the relationship between intelligence (fluid and crystallized) and creativity. Researchers imply that intelligence forms the basis for developing creativity [7].

In the literature there are suggestions that in some cases high intelligence quotient may hamper the performance of particular tasks. A broader perspective on intelligence as an ability to adapt, and simultaneously the realisation of how significant emotions are in dealing with difficult situations (including particular sports situations) make it possible to increase the importance of emotional intelligence. Emotional intelligence is a meta-ability. It is a complex of instrumental and motivational abilities that make emotions and emotional knowledge optimally available. They are responsible for its proper processing, noticing, identifying and understanding as well as regulating emotions. As a result, it enables humans to effectively utilize their own potential $[8,9,10]$. Research shows that emotional intelligence may be a factor contributing to top performance. It makes it possible to fully control the interaction between emotional and cognitive spheres $[11,12]$.

Creative attitude is not a popular research area in terms of sport. Nor is emotional intelligence, although this variable is a more common subject of interest for researchers mainly in the context of specificity, professional tasks, requirements, work efficiency and effective leadership of coaches [12, 13, 14]. It seems, though, that it is worthwhile to analyse these two vari- 
ables among athletes and compare them with untrained individuals. Young wrestlers are an interesting study group, all the more since the authors of this study have not found similar investigations in the literature.

The specificity of wrestling requires high level of physical fitness and proper personality traits on the part of wrestlers. The result of the sports fight depends to a large extent upon mental strength $[15,16]$. Literature reviews as well as observations allow us to assume that emotional intelligence and creative attitude (together with its components) may indirectly and directly increase mental strength. These features determine the skills required to solve new problems and adapt to changing situations, which may greatly influence the effectiveness of sports task performance during considerable physical exertion and fatigue.

In the course of the training process wrestlers undergo an intensive training in social skills. They need competencies that are indispensable for dealing with various situations encountered in sport. Without optimal levels of emotional intelligence or creative attitude it would be more difficult to make appropriate decisions, perform proper activities, perceive cause-andeffect relationships in sports training or avoid wrong actions and inappropriate ways of solving problems $[15,16]$. It is useful not only in motoric task learning or proper functioning in training and competition circumstances but also, owing to adequate skills transfer, in terms of effective and satisfying functioning in other areas of life [17].

Women have taken up wrestling only recently. Even though women's wrestling is a relatively new sport [18, 19], female wrestlers already participate in major competitions including the Olympic Games [20]. As in the case of men's wrestling, there are many factors that contribute to better performance and, eventually, to overcoming opponents. A female wrestler needs to demonstrate adequate abilities and high levels of motoric and technical-tactical preparation as well as optimal mental preparation [21]. According to the authors, it is also necessary to develop sport-specific emotional intelligence and creative attitude.

The specificity of creative attitude and emotional intelligence as well as their significance for human development has become the conceptual framework of research presented in this work. The aim of the study was to investigate these variables among young people (on the threshold of adulthood) with various interests and hobbies - female and male wrestlers and untrained female and male students ${ }^{1}$.

There is some indication that the culture influences development of creative attitude $[3,22]$. Bearing in mind the specificity of organisational culture of sports environment and the system of training for children and youth, it may be assumed that differences may occur between the groups participating in this research.

\section{Material and methods}

Seventy-eight subjects participated in the study. They were divided into four groups. The first group included girls $(n=20)$ from the regional of Lublin wrestling team of cadets and juniors. They were $16.9 \pm 0.99$ years of age and their training experience ranged from 3 to 8 years $(5.47 \pm 1.48)$. The second group consisted of untrained girls $(n=20)$ aged $16.9 \pm 0.22$. The third group included male wrestlers from the sports school of GrecoRoman wrestling $(n=19)$. They were $17.5 \pm 1.07$ years old and their training experience was between 3 and 9 years $(6.31 \pm 1.83)$. The fourth group included untrained boys $(n=19)$ aged $17.0 \pm 0.33$.

The first stage of research took place at a sports camp and it included a non-random sample of male and female wrestlers who were selected based on their age and performance level. The second stage was conducted in an upper-secondary school among randomly selected students.

The youth from the control groups filled in the same research form as the wrestlers; however, their form also included a question concerning interests and ways of spending leisure time. From amongst various options prepared on the basis of literature regarding leisure time [23] the subjects were requested to choose three activities and list them in the order of importance. This enabled us to choose those students who, in terms of sport, were neither active (no participation in sports activities) nor passive (no interest in media coverage concerning sports). Afterwards, 19 boys and 20 girls were randomly selected from this group. Their results were compared with the results of male and female wrestlers.

The following three research tools were employed in the study:

- a survey questionnaire - the authors' own questionnaire that made it possible to gather basic information also contained the list of free-time activities. This allowed us to select untrained students.

- the INTE questionnaire used to measure emotional intelligence, i.e. the ability to recognise emotions and use them in order to increase the effectiveness of acting and thinking [9];

- the KANH creative behaviour questionnaire (version I) for upper-secondary and university students; the questionnaire made it possible to assess creative vs. reproductive attitude together with their components:

- Cr scale - creative attitude (including $\mathrm{N}$ and $\mathrm{H}$ scales):

- N - nonconformity - "independence, activity, vitalism, adaptive flexibility, originality, persistence, courage, dominance, self-reliance, self-organisation, spontaneity, expressiveness, openness, resilience, responsibility, self-criticism, tolerance, high self-esteem" [5],

- H - heuristic behaviour/thinking - "independent observation, logical memory, productive imagination, divergent thinking, reconstructive and independent learning, learning through reasoning, intellectual flexibility, cognitive activity, reflexivity, intellectual independence, constructive and verbal creativity, (...) potential abilities regarding artistic creativity" [5],

- Re scale - reproductive attitude (including $\mathrm{K}$ and $\mathrm{A}$ scales):

- K - conformity - "dependence, passivity, lack of adaptive flexibility, stereotypicality, submissiveness, weakness, timidity, lack of independence, internal disorganisation, excessive inhibition, defensiveness, low resilience and persistence, irresponsibility, lack of criticism, intolerance, low self-esteem" [5],

- A - algorithmic behaviour/thinking - "copying and reproductive attitude"; "controlled perceptiveness, mechanical memory, reproductive imagination, convergent thinking, reproductive and oriented learning limited to understanding and logical analysis, lack of intellectual flexibility, cognitive passivity, low level of reflexivity, tendency to imprinting, low level of processing and creating abilities, lack of technical and artistic ingenuity" [5].

\footnotetext{
${ }^{1}$ The term "female and male students" refers to young people with various interests and hobbies who do not practise any sports. The authors are aware of the fact that female and male wrestlers participating in the study are also students.

${ }^{2}$ Training experience of female and male wrestlers was compared with the use of the non-parametric Mann-Whitney U test; no significant differences were noted $\mathrm{U}=137.50, \mathrm{p}=0.13$.
} 
It is worth highlighting that "there is a theoretical basis for analysing the structure of creative behaviour as creative attitude" [5]. Therefore, in this work the terms of "creative attitude" and "creative behaviour" are used interchangeably.

\section{Results}

For each scale of the KANH and INTE questionnaires the raw scores (RS) and the test scores (TS, according to sten norms for this age group provided by the authors of the tools) were calculated. Table no. 1 shows mean scores (with standard deviation) for each group of wrestlers and untrained individuals. Due to the number of subjects, the scores were compared with the use of the non-parametric Mann-Whitney U test. The table illustrates test values and levels of significance.

In the group of untrained subjects female students demonstrated higher levels of emotional intelligence and conformity than their male counterparts. They also manifested higher levels of emotional intelligence, nonconformity, heuristic thinking and creative attitude than female wrestlers. Female wrestlers demonstrated higher levels of conformity and reproductive attitude than female students.

Table no. 1 also shows $U$ test values that may indicate (in the case of groups with a low number of subjects) some tendency $(\mathrm{p} \leq 0.10)$. In turn, male wrestlers manifested lower levels of emotional intelligence and heuristic behaviour/thinking than untrained male students. Compared to male wrestlers, female wrestlers manifested higher levels of reproductive behaviour and attitude; however, the difference concerned raw scores only.

A vast majority of test scores fell into a range of medium scores with the exception of female wrestlers whose test scores achieved with the use of the INTE questionnaire pointed to low levels of emotional intelligence. Therefore, it is advisable to analyse test scores (TS) according to the following categories: low (1-4 stens), medium (5-6) and high (7-10) scores. The following figures illustrate detailed distributions. The symbols used refer to proper groups and gender of the subjects: FW female wrestlers, FS - female students (Fig. 1), MW - male wrestlers, MS - male students. The detailed illustration of acronyms can be found in the key section (Fig. 2).

The differences observed between mean scores of female subjects are illustrated in the following distributions. The distribution of the categories of scores obtained within the scales of INTE $\left(\mathrm{chi}^{2}=16.18, \mathrm{p} \leq 0.01\right), \mathrm{K}\left(\mathrm{Chi}^{2}=8.57 \mathrm{p} \leq 0.01\right)$ and $\mathrm{Cr}$ $\left(\mathrm{chi}^{2}=10.20, \mathrm{p} \leq 0.01\right)$ differentiates the groups of female wrestlers vs. female students. Half of the female students demonstrated a high level of emotional intelligence, while in the group of female wrestlers it was manifested by one subject.

Table 1. Comparison of scores obtained using the INTE and KANH questionnaires ${ }^{1}$

\begin{tabular}{|c|c|c|c|c|c|c|c|c|}
\hline \multirow{2}{*}{ Scale } & \multicolumn{4}{|c|}{$x \pm S D$} & \multicolumn{4}{|c|}{ Mann.Whitney U test } \\
\hline & female wrestlers & female students & male wrestlers & male students & $\begin{array}{c}\text { female wrestlers- } \\
\text { male wrestlers }\end{array}$ & $\begin{array}{l}\text { female students- } \\
\text { male students }\end{array}$ & $\begin{array}{l}\text { female wrestlers- } \\
\text { female students }\end{array}$ & $\begin{array}{l}\text { male wrestlers- } \\
\text { male students }\end{array}$ \\
\hline INTE - RS & $115.5 \pm 12.8$ & $135.0 \pm 13.3$ & $112.7 \pm 18.3$ & $121.2 \pm 12.6$ & $\cdot$ & $\begin{array}{l}88.5 \\
0.00 \ldots\end{array}$ & $\begin{array}{c}48.0 \\
0.00 \ldots\end{array}$ & $\begin{array}{l}124.5 \\
0.10\end{array}$ \\
\hline INTE - TS & $3.70 \pm 1.45$ & $6.75 \pm 2.15$ & $4.26 \pm 2.13$ & $5.26 \pm 1.73$ & $\cdot$ & $\begin{array}{l}114.0 \\
0.03\end{array}$ & $\begin{array}{c}49.0 \\
0.00 \ldots\end{array}$ & $\begin{array}{l}124.5 \\
0.10\end{array}$ \\
\hline $\mathbf{K}-\mathbf{R S}$ & $15.3 \pm 3.96$ & $12.1 \pm 4.30$ & $13.0 \pm 6.12$ & $14.2 \pm 3.99$ & - & $\begin{array}{c}89.5 \\
0.00 \ldots\end{array}$ & $\begin{array}{c}93.5 \\
0.00 \ldots\end{array}$ & $\cdot$ \\
\hline$K-T S$ & $6.50 \pm 1.54$ & $5.35 \pm 1.53$ & $5.74 \pm 2.56$ & $6.05 \pm 1.71$ & - & $\begin{array}{l}115.5 \\
0.03\end{array}$ & $\begin{array}{c}106.0 \\
0.01 \\
\end{array}$ & - \\
\hline A - RS & $17.4 \pm 3.39$ & $16.0 \pm 3.01$ & $15.3 \pm 4.10$ & $16.1 \pm 2.27$ & $\begin{array}{l}125.5 \\
0.07 \\
\end{array}$ & $\cdot$ & $\cdot$ & $\cdot$ \\
\hline A - TS & $6.95 \pm 1.79$ & $6.20 \pm 1.58$ & $5.95 \pm 2.09$ & $6.16 \pm 1.21$ & - & $\cdot$ & . & - \\
\hline $\mathbf{N}$ - RS & $17.7 \pm 3.54$ & $19.8 \pm 3.11$ & $18.5 \pm 4.88$ & $18.3 \pm 4.01$ & - & - & $\begin{array}{l}130.5 \\
0.06 \\
\end{array}$ & - \\
\hline $\mathbf{N}-\mathbf{T S}$ & $4.75 \pm 2.00$ & $6.05 \pm 1.79$ & $5.26 \pm 2.28$ & $5.21 \pm 2.27$ & $\cdot$ & - & $\begin{array}{l}123.5 \\
0.04\end{array}$ & $\cdot$ \\
\hline H - RS & $15.5 \pm 5.05$ & $18.3 \pm 2.57$ & $17.5 \pm 4.03$ & $19.6 \pm 3.61$ & $\cdot$ & $\cdot$ & $\begin{array}{l}113.5 \\
0.02\end{array}$ & $\begin{array}{l}124.0 \\
0.10\end{array}$ \\
\hline H - TS & $4.65 \pm 2.03$ & $5.85 \pm 1.35$ & $5.42 \pm 2.09$ & $6.52 \pm 1.80$ & $\cdot$ & $\cdot$ & $\begin{array}{c}120.5 \\
0.03 \\
\end{array}$ & $\begin{array}{l}119.0 \\
0.07 \\
\end{array}$ \\
\hline $\mathrm{Cr}-\mathrm{RS}$ & $33.2 \pm 7.86$ & $37.9 \pm 4.08$ & $36.1 \pm 8.28$ & $37.8 \pm 6.78$ & - & $\cdot$ & $\begin{array}{c}112.0 \\
0.02 \\
\end{array}$ & - \\
\hline $\mathrm{Cr}-\mathrm{TS}$ & $4.70 \pm 2.05$ & $5.85 \pm 1.09$ & $5.26 \pm 2.30$ & $5.74 \pm 1.88$ & - & - & $\begin{array}{c}121.0 \\
0.03 \\
\end{array}$ & $\cdot$ \\
\hline $\mathrm{Re}-\mathrm{RS}$ & $32.7 \pm 6.73$ & $28.1 \pm 5.97$ & $28.3 \pm 9.15$ & $30.3 \pm 5.62$ & $\begin{array}{l}129.5 \\
0.09 \\
\end{array}$ & . & $\begin{array}{c}118.0 \\
0.03 \\
\end{array}$ & . \\
\hline $\operatorname{Re}-\mathrm{TS}$ & $6.65 \pm 1.78$ & $5.55 \pm 1.53$ & $5.84 \pm 2.31$ & $6.05 \pm 1.43$ & - & . & $\begin{array}{l}129.0 \\
0.06\end{array}$ & - \\
\hline
\end{tabular}

${ }^{1}$ Key:

- statistically significant values are presented in grey table cells; RS - raw scores, TS - test scores; : INTE - scores obtained with the use of the INTE questionnaire (emotional intelligence), $\mathrm{K}$ - scale K scores (conformity) of the KANH questionnaire, A - scale A scores (algorithmic behaviour/thinking) of the KANH questionnaire, $\mathrm{N}$ - scale $\mathrm{N}$ scores (nonconformity) of the KANH questionnaire, $\mathrm{H}$ (heuristic behaviour/thinking) - scale $\mathrm{H}$ scores of the KANH questionnaire, Cr - scale Cr scores (creative attitude) of the KANH questionnaire, Re - scale Re scores (reproductive attitude) of the KANH questionnaire. 
Almost half of the surveyed sportswomen revealed high levels of conformity, whereas a vast majority of female students (over $90 \%)$ obtained low and medium levels. Half of the female wrestlers demonstrated low levels of creative attitude, while similar scores were achieved by one female student.

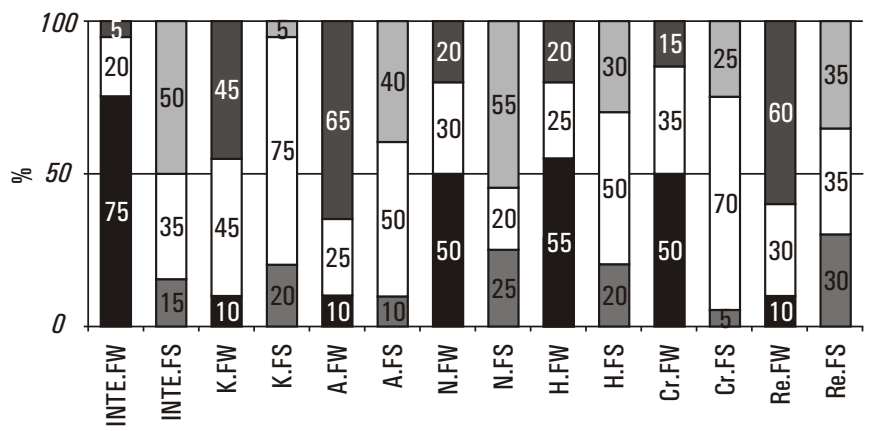

$$
\begin{array}{|l}
\hline \text { high } \quad \square \text { medium } \quad \square \text { low } \\
\hline
\end{array}
$$

Figure 1. Comparison of categories of scores within particular scales (females)

Illustration of acronyms and abbreviations:

- research methods employed: INTE - scores obtained with the use of the INTE questionnaire (emotional intelligence), $\mathrm{K}$ - scale $\mathrm{K}$ scores (conformity) of the KANH questionnaire, A - scale A scores (algorithmic behaviour/thinking) of the KANH questionnaire, $\mathrm{N}$ - scale $\mathrm{N}$ scores (nonconformity) of the KANH questionnaire, $\mathrm{H}$ (heuristic behaviour/thinking) - scale $\mathrm{H}$ scores of the KANH questionnaire, $\mathrm{Cr}$ - scale $\mathrm{Cr}$ scores (creative attitude) of the KANH questionnaire, $\mathrm{Re}$ - scale Re scores (reproductive attitude) of the KANH questionnaire, - groups under investigation (acronyms): W - student wrestlers, S - untrained students, $\mathrm{F}$ - females, $\mathrm{M}$ - males.

Contrary to the findings regarding the girls, a parallel comparison of the categories of scores in the group of boys did not reveal significant differences.

The analysis of the values of correlations between variables revealed a number of significant relationships. A comparison of statistically significant indices is depicted in the following table.
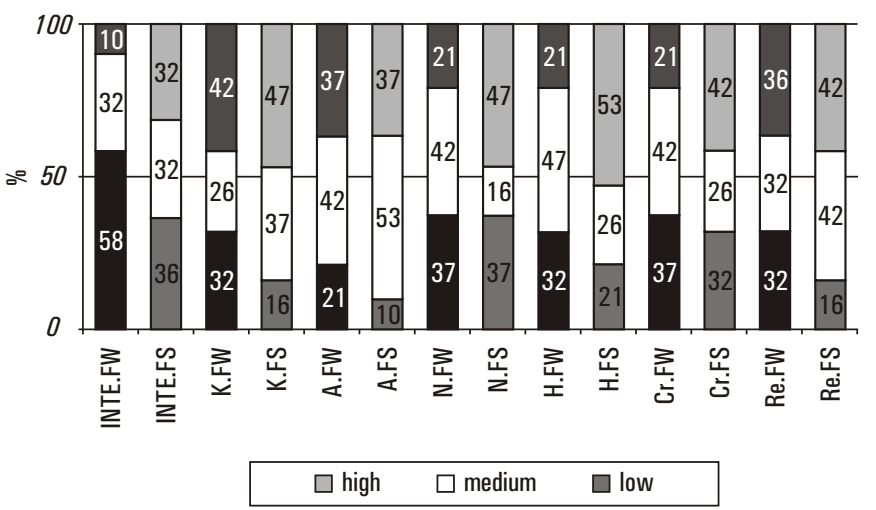

Figure 2. Comparison of categories of scores within particular scales (males) ${ }^{2}$

Illustration of acronyms and abbreviations:

- research methods employed: INTE - scores obtained with the use of the INTE questionnaire (emotional intelligence), $\mathrm{K}$ - scale $\mathrm{K}$ scores (conformity) of the KANH questionnaire, A - scale A scores (algorithmic behaviour/thinking) of the KANH questionnaire, $\mathrm{N}$ - scale $\mathrm{N}$ scores (nonconformity) of the KANH

questionnaire, $\mathrm{H}$ (heuristic behaviour/thinking) - scale H scores of the KANH questionnaire, $\mathrm{Cr}$ - scale $\mathrm{Cr}$ scores (creative attitude) of the KANH questionnaire, $\mathrm{Re}$ - scale Re scores (reproductive attitude) of the KANH questionnaire,

- groups under investigation (acronyms): W - student wrestlers, S - untrained students, $\mathrm{F}$ - females, $\mathrm{M}$ - males.

In each of the examined groups numerous intercorrelations between the scales of the KANH questionnaire were observed. Creative attitude is connected with its components, as is the case with reproductive attitude. Moreover, the analyses carried out on female and male wrestlers revealed numerous relationships between creative and reproductive attitude. In those groups and in the group of male students no relationships between the levels of emotional intelligence and selected aspects of creativity were noted. It was only in the group of female students that emotional intelligence was connected with creative attitude and its component, i.e. nonconformity.

Table 2. Significant values of score correlations from particular scales ${ }^{1}$

\begin{tabular}{|c|c|c|c|c|c|c|c|c|c|c|c|c|c|c|c|c|}
\hline \multirow{2}{*}{\begin{tabular}{l} 
Spearman's \\
rho \\
\multirow{2}{c}{$\infty$}
\end{tabular}} & \multicolumn{7}{|c|}{ GIRLS } & \multirow{2}{*}{$\begin{array}{l}\text { scale } \\
\text { INTE }\end{array}$} & \multicolumn{7}{|c|}{ BOYS } & \multirow{8}{*}{ 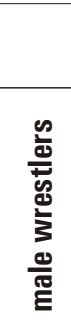 } \\
\hline & $X$ & & & & & & & & $X$ & & & & & & & \\
\hline \multirow{6}{*}{ 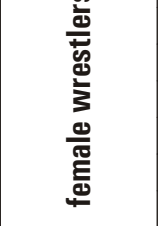 } & . & $X$ & & & & & & K & . & $X$ & & & & & & \\
\hline & . & $.70^{* *}$ & $X$ & & & & & A & - & $.59^{* *}$ & $X$ & & & & & \\
\hline & . & $.65^{* *}$ & $.79 * *$ & $X$ & & & & $\mathbf{N}$ & . & . & $.61^{* *}$ & $X$ & & & & \\
\hline & . & $.64^{* *}$ & - & $.56^{*}$ & $X$ & & & H & . & . & $.61^{* *}$ & $.64^{* *}$ & $X$ & & & \\
\hline & . & $.73^{* *}$ & $.67^{* *}$ & $.85^{* *}$ & $.88^{*}$ & $X$ & & $\mathrm{Cr}$ & . & . & $.66^{* *}$ & $.90^{* *}$ & $.88^{* *}$ & $X$ & & \\
\hline & $\cdot$ & $.96^{* *}$ & $.86^{* *}$ & $.74^{* *}$ & $.59 * *$ & $.76^{* *}$ & $X$ & $\mathbf{R e}$ & $\cdot$ & $.94^{* *}$ & $.81^{* *}$ & $.56^{*}$ & . & $.61^{* *}$ & $x$ & \\
\hline scale & INTE & K & A & $\mathbf{N}$ & H & $\mathrm{Cr}$ & Re & scale & INTE & K & A & $\mathbf{N}$ & H & $\mathrm{Cr}$ & Re & scale \\
\hline \multirow{7}{*}{ 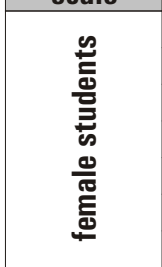 } & $X$ & & & & & & & INTE & $X$ & & & & & & & \multirow{7}{*}{ 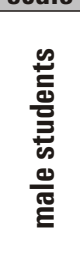 } \\
\hline & - & $X$ & & & & & & K & . & $X$ & & & & & & \\
\hline & $\cdot$ & $.58^{* *}$ & $X$ & & & & & A & - & $.74^{* *}$ & $X$ & & & & & \\
\hline & $.47^{*}$ & $\cdot$ & - & $X$ & & & & $\mathbf{N}$ & - & $\cdot$ & $\cdot$ & $X$ & & & & \\
\hline & . & . & - & . & $X$ & & & H & . & . & . & $.59^{* *}$ & $X$ & & & \\
\hline & $.58^{* *}$ & $\cdot$ & $.54^{*}$ & $.76^{* *}$ & $.55^{*}$ & $X$ & & $\mathrm{Cr}$ & . & $\cdot$ & $\cdot$ & $.85^{* *}$ & $.90^{* *}$ & $X$ & & \\
\hline & . & $.90^{* *}$ & $.79 * *$ & . & . & 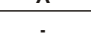 & $X$ & $\mathrm{Re}$ & & $.91^{* *}$ & $.92^{* *}$ & - & . & . & $X$ & \\
\hline
\end{tabular}

${ }^{1}$ Key:

- INTE - scores obtained with the use of the INTE questionnaire (emotional intelligence), K - scale K scores (conformity) of the KANH questionnaire, A - scale A scores (algorithmic behaviour/thinking) of the KANH questionnaire, $\mathrm{N}$ - scale $\mathrm{N}$ scores (nonconformity) of the KANH questionnaire, $\mathrm{H}$ (heuristic behaviour/thinking) - scale $\mathrm{H}$ scores of the KANH questionnaire, $\mathrm{Cr}$ - scale Cr scores (creative attitude) of the KANH questionnaire, Re - scale Re scores (reproductive attitude) of the KANH questionnaire. 


\section{Discussion}

In the course of the analyses regarding relationships between variables some regularity was observed. Although intercorrelations between the scales of the KANH questionnaire in female and male students concerned specific types of attitude and their components, in female and male wrestlers relations between creative attitude and reproductive attitude as well as between their components were found. It may be connected with the specificity of sports training and wrestling. Only when wrestlers are capable of reproducing the elements they have learnt will they be able to make creative use of them. However, some clearly defined rules must be always taken into consideration. Years of training and the necessity to follow strict sports routine may have led to the development of the types of behaviour/attitude other than those observed in their untrained counterparts. It is also possible that people with this type of a psychological profile were picked already at the stage of initial selection. It seems further research regarding this idea might be worthwhile.

Perhaps in the training of wrestlers not enough emphasis was placed on developing a sense of locus of control and selfefficacy as well as types of creative attitude, while the methods applied have not contributed to the development of those psychological resources. The cause may also be found in initial stages of selection, as due to the fact that wrestling, particularly women's wrestling, is a niche sport, these training activities may not have been performed properly.

An interesting thing is the lack of relationship (in most groups) between the components of creative/reproductive attitude and emotional intelligence. It was only in the group of female students, who demonstrated the highest levels of emotional intelligence, that the relationship between this feature and creative attitude together with its component, i.e. nonconformity, was observed. It is possible that higher levels of emotional intelligence form the basis for creative behaviour, including nonconformity. This kind of relationship would be promising. Therefore, it is worth developing these features and enhancing them correlatively as well as simultaneously emphasising an ethical aspect of attitudes that are currently demonstrated. Apart from unquestionable positive consequences of creativity there is also its "dark side". More creative individuals are more likely to manifest dishonest patterns of behaviour [24]. Strengthening empathy, which is a component of emotional intelligence, might be an interesting solution.

It is worth determining the levels of creative attitude among youth, i.e. in a period between childhood and adulthood. Creativity observed in children and adults has different images, aims, manifestations and applications. In an educational process it is worth developing desired elements of creativity and not just teaching ready-made solutions applied in difficult adult life situations [25]. Creative attitude and emotional intelligence may also become important psychological resources that will turn out to be useful in developing diverse talents and transforming particular skills (acquired at school) into competencies (useful, inter alia, while studying and in a future professional career). They will form a crucial element of mental (emotional) resilience in periods of intensive life changes [26].

Therefore, teachers and coaches ought to combine directed and consistent educational actions in order to enhance the potential of students and athletes. Various kinds of training oriented at creativity and psychological skills, coaching and mentoring elements and, first and foremost, creating an emotionally healthy atmosphere of development may be the right ways to support talents both at school and in sport. Sport in particular requires holistic strengthening of potential so that athletes can face various challenges in any area in an optimal way both during and after their sports careers [17]. Emotional intelligence is a variable that is definitely worth strengthening. In a sense it is a universal feature, as it guarantees personal success in various areas. Moreover, it is believed to have a direct influence on mental well-being [11].

However, it is intriguing that the authors' own investigations regarding female and male wrestlers pointed to their medium or low levels of emotional intelligence and creative attitude together with its components. They ought to serve as guidelines for working with children and youths, for those who create a sports environment, especially for coaches and sports psychologists. Close attention should be paid to designing activities aimed at female wrestlers. The number of differences observed in this group (compared to their untrained counterparts) ought to become a subject of discussion of the problems connected with the specificity of female sports - particularly combat sports, which are regarded as masculine.

As early as in the primeval times, physical fitness was essential mainly for men for hunting, defence and military purposes. At that time they performed activities connected with a direct combat with an enemy. The fundamentals of wrestling were formed then. In ancient times wrestling (associated with physical fitness and violent combats) was exclusively for men. It required enormous strength, bravery and stamina that was often accompanied by pain and suffering. Due to its specificity, it was viewed as men's domain. The image of wrestling was contradictory to the image of women, who were perceived as vulnerable, sensitive and beautiful. Recently, however, wrestling has been made available to women. Tackling their opponents, they flirt with the audience using their grace and powerful emotions. In this way they have taken wrestling shows to a different level.

In general, sport "remains an island for men where only a certain number of women are allowed, but only those who are willing to abide by rules established by men for men" [27]. Despite social transformations (also in wrestling) and changes regarding social roles, the specificity of sport is connected with the necessity (also for women) to conform to a stereotype of a winner, a hero - a man. Obviously, it may lead to their fear of success, which is enhanced by typical sports fears of failure [27, 28].

Although psychological factors are not treated as primary determinants of motoric learning, it is worth noting that their role (along with neurophysiological and anatomical factors) is significant. In particular, emotional and intellectual factors are growing in importance [29]. The review of the literature allows us to assume that creative attitude and emotional intelligence may exert an influence on the course of motoric learning (in wrestling as well). Therefore, it is worth conducting further research on the role of those variables also in the process of movement learning.

The authors' own research results may also be useful for teachers and coaches, who aim not only at fulfilling basic teaching requirements and achieving particular educational or sports goals but also at supporting the development of skills that enable young people to adapt to the ever-changing reality. Creative vs. reproductive attitude and emotional intelligence seem to be important elements that ensure personal success in various areas: sport, education, personal life, etc.

In general, the authors' own research results revealed medium levels of emotional intelligence and creative vs. reproductive attitude among the youth under investigation. It was only in the group of female wrestlers that the levels of emotional intelligence were low. This variable, as well as the levels of nonconformity, heuristic behaviour/thinking and creative attitude were significantly lower among female wrestlers than among untrained girls. Compared to female students, female wrestlers manifested higher levels of conformity and reproductive attitude. In turn, female students demonstrated higher levels of emotional intelligence and conformity than their male counter- 
parts. No significant differences (just tendencies) were observed when comparing female and male wrestlers.

The authors' own research results indicate the necessity to carry out longitudinal research on untrained individuals and athletes mainly in the area of sport for children and youth. Such a diagnosis will make it possible to profile various educational effects (especially in sports psychology), thus leading to the strengthening of potential.

\section{Conclusions}

The majority of subjects demonstrated medium levels of emotional intelligence and creative vs. reproductive attitude. Only female wrestlers manifested low levels of emotional intelligence; their levels of nonconformity, heuristic thinking and creative attitude were also lower than in female students. However, female wrestlers revealed higher levels of conformity and reproductive attitude. In turn, female students manifested higher levels of emotional intelligence and conformity than male students. No differences were found between male and female wrestlers.

The findings show that it is necessary to conduct longitudinal research on athletes and untrained individuals. The authors suggest that the relationship between selected psychological resources and the effectiveness of motoric skill learning should be verified.

The authors' own research results as well as further (planned) investigations will make it possible to profile various educational effects, thus leading to the mental enhancement of potential in young wrestlers.

\section{Literature}

1. Nęcka, E. (2000). Creativity. In J. Strelau (Ed.). Psychology. Academic textbook Vol.II (pp., 783-807). Gdańsk: GWP. [in Polish]

2. Nęcka, E. (2002). Psychology of creativity. Gdańsk: GWP. [in Polish]

3. Tahereh, R. \& Mahnoush S. (2012). Creativity. Australian Journal of Basic and Applied Sciences 6(2), 25-28.

4. Cudowska, A. (2004). The formation of creative attitude in the process of education. Białystok: Trans Humana. [in Polish]

5. Popek, S. (2000). Creative Behaviour Questionnaire-KANH. Lublin: UMCS. [in Polish]

6. Popek, S., Bernacka E. \& Lickiewicz J. (2005). Creative Behaviour Questionnaire KANH III as a method of diagnosing creative attitude of a manager. Education of economists and managers (pp. 1, 35-48). Warszawa: SGH. [in Polish]

7. Nusbaum, E. \& Silvia P. (2011). Are intelligence and creativity really so different? Fluid intelligence, executive processes, and strategy use in divergent thinking. Intelligence 39(1), 36-45.

8. Goleman, D. (1997). Emotional intelligence. Poznań: Media Rodzina. [in Polish]

9. Jaworowska, A. \& Matczak A. (2001). Emotional Intelligence Questionnaire. Textbook. Warszawa: Pracownia Testów Psychologicznych PTP. [in Polish]

10. Salovey, P. \& Sluyter D. (Eds.) (1999). Emotional development vs. emotional intelligence. Educational problems. Poznań: Dom Wydawniczy Rebis. [in Polish]
11. Bar-on, R. (1999). The emotional question inventory (EQ-I). A measure of emotional intelligence. Toronto, Canada: Multihealth systems. Handbook of emotional intelligence. San Francisco, USA: Jossey- Bass.

12. Chan, J.T. \& Mallett C.J. (2011). The Value of Emotional Intelligence for High Performance Coaching. International Journal of Sports Science and Coaching 6(3), 315-328.

13. Afkhami, E., Shirmohammadzadeh M., Moghaddam J.B., Aghdam J.K. \& Salehian M.H. (2012). Emotional Intelligence and Coaching Efficacy in Female Coaches. Annals of Biological Research 3(3), 1236-1241.

14. Rutkowska, K. \& Gierczuk D. (2012). Emotional intelligence and the sense of efficiency of coaching and instructing in wrestling. Polish Journal of Sport and Tourism 19(1), 46-57.

15. Petrov, R. (1987). Freestyle and Greco-roman wrestling. Lausanne, Switzerland: FILA.

16. Hendrix, B. (1996). Wrestle to Win. Marietta, GA, USA: High Performance Athletics.

17. Petitpas, A., Champagne D., Chartrand J., Danish S. \& Murphy S. (1997). Athlete's guide to career planning. Keys to success from the playing field to professional life. Champaign, Human Kinetics.

18. Payette, M. (1992). Women's wrestling. Canadian Wrestler 13, 3-5.

19. Allen, L. (2000). Girls wrestling, where is it going? Wrestling USA 35, 26-28.

20. Popke, M. (2004). Welcome mat: the Olympic debut of women's wrestling could jump-start the sport at the prep level. Athletic business 28, 30-33.

21. Shi, H. \& Ma Z. (2007). Research on elite female wrestlers' psychological stress control before competitions. Journal of Beijing University of Physical Education 30, 1049-1050.

22. Erez, M. \& Nouri R. (2010). Creativity: the influence of cultural, social, and work contexts. Management and Organization Review 6(3), 351-270.

23. Pięta, J. (2008). Pedagogy of Spare Time. Warszawa: WSE. [in Polish]

24. Gino, F. \& Ariely D. (2012). The dark side of creativity: original thinkers can be more dishonest. Journal of Personality and Social Psychology 102(3), 445-459.

25. Kudryavtsev, V. (2011). The phenomenon of child creativity. International Journal of Early Years Education 19(1), 45-53.

26. Subotnik, R., Edmiston A., Cook L. \& Ross M. (2010). Mentoring for talent development, creativity, social skills, and insider knowledge: The APA Catalyst Program. Journal of Advanced Academics 21(4), 714-739.

27. Mikołajczyk, M. (2003). Does sport have to have a gender? In J. Kłodecka-Różalska (Ed.) Sportswoman - a woman of success. Advantages and obstacles in women's sports activity (pp. 24-42). Warszawa: PSSK, IS. [in Polish]

28. Pospiszyl, K. (1993). Is a lady afraid of success? Trening 4, 83-89. [in Polish]

29. Petryński, W. \& Feigenberg J.M. (2009). Emotional factors in motoric control in humans. Antropomotoryka 47, 77-86.

Submitted: September 10, 2012

Accepted: October 22, 2012 